\title{
3-Parameter Hough Ellipse Detection Algorithm for Accurate Location of Human Eyes
}

\author{
Qiufen Yang \\ School of Information Science and Engineering, Central South University, Changsha, China \\ Email: yqf5569@sohu.com
}

Huosheng $\mathrm{Hu}$

School of Computer Science \& Electronic Engineering, University of Essex, Colchester CO4 3SQ, UK

Email: hhu@essex.ac.uk

Weihua Gui

School of Information Science and Engineering, Central South University, Changsha, China

Email:whgui@csu.edu.cn

Shuren Zhou

Computer \& Communication Engineering School, Changsha University of Science \& Technology, Changsha, China Email: zsr@ csust.edu.cn

Can Zhu

School of Traffic and Transportation Engineering, Changsha University of Science \& Technology, Changsha, China

Email: zhc@csust.edu.cn

\begin{abstract}
Accurately positioning the Human Eyes plays an important role in the detection of the fatigue driving. In order to improve the performance of positioning of human face and eyes, an accurately positioning method of the human eyes is proposed based on the 3-parameter Hough ellipse detection. Firstly, the human face area is divided by using the skin color clustering and segmentation algorithm. Then, the segmented image is filtered by using its geometric structure and the approximate positions of the human face area and eyes are calculated. Finally, on the basis of the spinning cone-shape eye model, the position of human face and eyes is accurately determined by using the 3-parameter Hough transformation ellipse detection algorithm. The different images of human face are used to test the performance of the proposed method. The experimental results show that the extreme value of upper and lower eyelids and the actual position is 0.104 and the proposed algorithm has higher positioning accuracy.
\end{abstract}

Index Terms-Skin Color Clustering; Positioning of Human Eyes; Hough Transformation; Ellipse Detection

\section{INTRODUCTION}

Along with rapid economic development, the number of automobiles in the whole globe has been constantly increasing; therefore, the problem of road safety becomes a hot issue. Besides, fatigue driving is one of the major reasons for traffic accidents. In order to solve this problem, we thus conduct researches about fatigue driving and place our emphasis on human eye tracking in detecting devices for fatigue driving as human eye tracking serves as an important step in drowsy driving detection system.

Domestic researches on fatigue driving have become more and more since 2003, focusing on the state information of the head or face of the drivers. In 2001, from the perspective of the image recognition technology, Zheng Pei et al. applied the PERCLOS fatigue parameters to develop the measurement system of motor driver fatigue (Li Zengyong and Wang Chengzhu, 2001). Wang Xiaojuan started the research on the combination of the eye state information and mouth state information(Cootes T F and Taylor C J, 1995), capturing the head position information through the mobile camera mounted on the platform, then finding the eyes and the mouth position in the image, and extracting and combining all the data information to determine fatigue.Therefore, it has important research value to use the camera to continuously observe the image of driver's facial features such as eyes, ears, nose and mouth and then determine whether the driver is fatigue driving in accordance with existing facial recognition technology. Federal Highway Administration uses the PERCLOS method, which determines the fatigue degree of eyes in accordance with the duration of eyes being closed during a certain period, and its precondition is the accurate positioning of human eyes. Therefore, it is particularly important to conduct accurate positioning of eyes in accordance with the color image sequences captured by the camera.

The positioning method of human eyes generally consists of two steps: the first step is coarse positioning, 
which is to find the possible spot of eyes on the image or initially determine the approximate position of human eyes; the second step is accurate positioning, which uses certain rules or verification method to determine the exact position of two eyes. At present, the positioning algorithms for human eyes include: the method proposed by Reinders et al. which uses the neural network and the micro characteristic of eyes to position the feature of human face [1], its shortage is that it requires a high calculated quantity for multi-scale detection of human eyes; Zhu et al. used the integral image to find the candidate points of pupil and then used SVM for detection [2], which seldom used the shape information of eyes; Liu et al. used the geometric characteristics of iris to detect human eyes, then conducted pairing in accordance with certain rules, and then used the neural network to conduct verification [3]; Bala et al. proposed a eye positioning method based on genetic algorithm and decision tree [4], which cannot effectively address the situation of face rotation, and it does not have adequate recognition ability of objects similar to human eyes; Feng and Yuen proposed a multi-thread method to position eyes [5]; Huang and Weshler used the SVM method to obtain the posture of human face and the position of human eyes [6]; Wang Shoujue et al. used the geometry complexity to rapidly position human eyes [7]; based on edge extraction, Zhang et al. used the Hough transformation [8] and circle detection algorithm to position the iris of eye, but the generated image is not necessarily consistent with the actual shape of eyes.

Therefore, all the accurate positioning algorithms for human eyes proposed up until now require a high calculated quantity, some are difficult to realize, and some are sensitive to the rotation, translation or size change of image, which are highly limited. In this paper, an innovative accurate positioning method for human eyes based on the color space of skin color clustering and the Hough transformation ellipse detection proposed. First of all, through the skin color clustering and segmentation algorithm, this method divides the human face area to obtain the binary image of human face; then, the skin color clustering and segmentation algorithm is used to conduct connectivity analysis of the binary image that includes the human face area, then, geometric filtration is conducted, and the centroid point of hole in the candidate human face area is calculated to find possible human eye pair; based on approximate detection of the human face area and eyes, the spinning cone-shape eye model is proposed to analyze the parameters of elliptical calculation, which overcomes the shortage of traditional 5-parameter space ellipse method, and the 3parameter space method is used; at last, the Hough transformation ellipse detection algorithm is used to conduct accurately positioning of human eyes. With the relative error scale of 0.104 , this algorithm can reach an accuracy of $100 \%$.

\section{COlOR Model of SkIN COlOR Clustering}

\section{A. Color Space of Skin Color Clustering}

In order to segment the human face area from the whole image, a reliable skin color model is built, which applies to various factors that affect the skin detection result, such as different skin colors, lighting conditions and covering.

The RGB space consists of the red, green and blue components, and because in this space, the color information and brightness information are mixed, while the difference of skin color is mainly caused by brightness, therefore, this color model is not suitable. Through statistics, we find that the $\mathrm{Cb}$ and $\mathrm{Cr}$ components present very stable clustering characteristics, as shown in Figure 1. By using the clustering between the chromaticity and brightness of skin [1] [2], the chromaticity and brightness components are selected to divide the $\mathrm{YCbCr}$ color space and conduct skin color modeling.
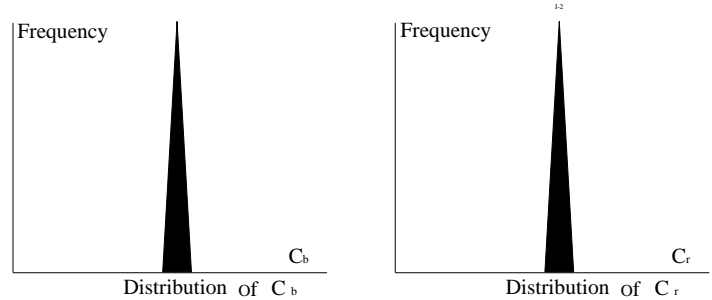

Figure 1. Distribution of $\mathrm{Cb}$ and $\mathrm{Cr}$ components in skin color

In Fig. 2, the distribution of gray level differences over images of eyes is shown. The gray level differences, calculated over different scales of skin, are shown in the Figure It can be seen that the distribution of gray level differences for the class of eye images is well approximated by the $\mathrm{YCbCr}$ color space. Further, it can be seen that the width $\mathrm{k}$ of the distribution increases with the scale.

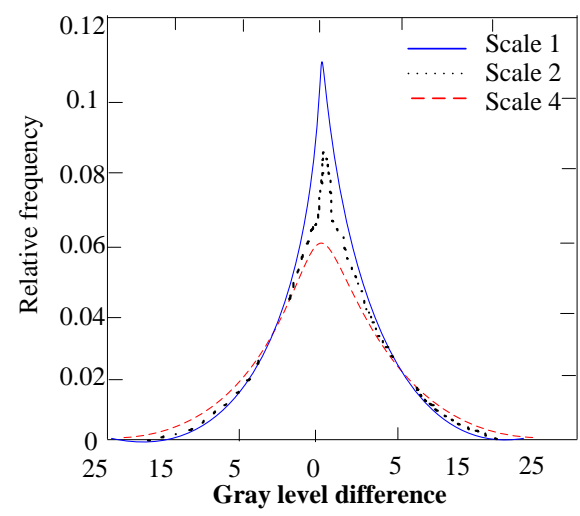

Figure 2. The distribution of gray level differences

\section{B. Gaussian Model of Skin Color Clustering}

After projecting the obtained facial image into the $\mathrm{YCbCr}$ space, the number of different $\mathrm{Cb}-\mathrm{Cr}$ pairs is calculated, the final count value is used as the vertical coordinate, and with $\mathrm{Cb}$ as the horizontal ordinate and $\mathrm{Cr}$ as the longitudinal coordinate, a three dimensional stereogram is drawn(Figure 3) [2]. 
For any pixel, its brightness component $\mathrm{Y}$ and the two chromaticity components $\mathrm{Cb}$ and $\mathrm{Cr}$ are statistically independent, which present Gaussian distribution. Because the brightness component $Y$ is susceptible to the change of light, therefore, only the two stable chromaticity components $\mathrm{Cb}$ and $\mathrm{Cr}$ are used to build the Gaussian model $G\left(m, V^{2}\right)$ :

$$
\begin{gathered}
m=(\overline{C r}, \overline{C b}) \\
\overline{C r}=\frac{1}{N} \sum_{i=1}^{N} C r \\
\overline{C b}=\frac{1}{N} \sum_{i=1}^{N} C b \\
V=\left[\begin{array}{ll}
\sigma_{c r, c r} & \sigma_{c r, c b} \\
\sigma_{c b, c r} & \sigma_{c b, c b}
\end{array}\right]
\end{gathered}
$$

In which, $\overline{C r}$ and $\overline{C b}$ are the corresponding mean values of $\overline{C r}$ and $\overline{C b}$, and $V$ is the covariance matrix.

Through the built skin color model, we transform a color image into a grayscale image, the grayscale value corresponds to the possibility of this point belonging to the skin area, and then, the grayscale image can be further transformed into a binary image by selecting appropriate threshold value, in which, 0 and 1 refer to the non-skin area and skin area respectively.

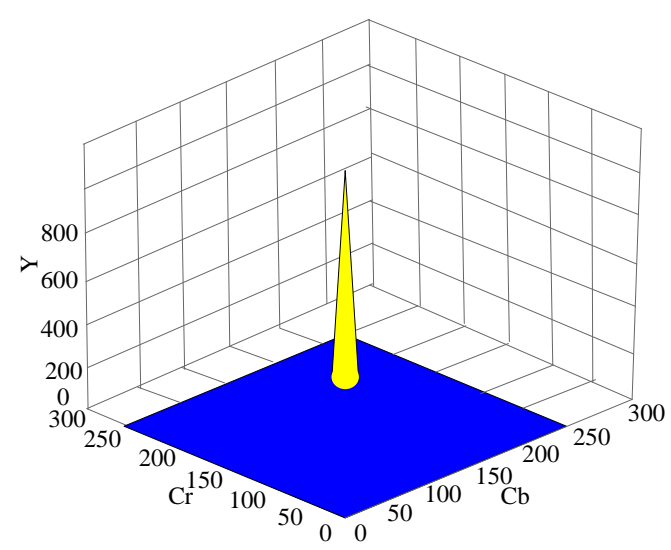

Figure 3. Distribution of skin color in the $\mathrm{YCbCr}$ space

\section{AREA SEGMENTATION DURING COARSE POSITIONING OF HUMAN EYES}

Because the human face is a connected area, we also conduct connectivity analysis of the binary image that includes the human face area obtained earlier in the color space of skin color clustering. For the noise existing in the inside and background of image, the $3 \times 3$ mid-value is adopted to conduct filtering and de-noising, and the expansion operator of morphology is used for filling to make the non-filled area as small as possible.

Based on the color segmentation algorithm proposed in the literature [12], the skin color clustering and segmentation algorithm is proposed, and the purpose of this algorithm is to ensure various clustering areas have consistent color.

The skin color clustering and segmentation algorithm is as the following:

(1) Divide the skin color image into $m \times n$ blocks, and calculate the mean value of skin color pixel $y_{i} C b_{i} C r_{i}$ of each block.

(2) Set the two-dimensional array clus to mark the clustering area of each block, and the initial value is set as 0 .

(3) Scan the image from top to bottom and left to right, find the first block with a clus value of 0 , use it as a new clustering area, then set clus as 1 , and initialize $\mathrm{Cb}, \mathrm{Cr}$ as well as the mean values of $\overline{C r}$ and $\overline{C b}$ of the area.

(4) Successively examine all skin color blocks with a clus value of 0 in the surrounding area of Block 8 , if its variance with the $\mathrm{Y}$ component of all blocks in current area is smaller than $\mathrm{c}_{1}, \frac{C b_{i} \overline{C b}+C r_{i} \overline{C r}}{\sqrt{C b_{i}^{2}+C r_{i}^{2}}+\sqrt{\left(\overline{\overline{C b})^{2}+(\overline{C r})^{2}}\right.}} \geq c_{2}$ it should be included into the area, the clus value should be set as 1 , and $\overline{C r}, \overline{C b}$ as well as the mean values of $\overline{C r}$ and $\overline{C b}$ should be recalculated; repeat (4) until all connected blocks are scanned.

(5) Record the searched area, return to Step (3), conduct a new round of search, and add 1 to the clus value of each block until the clus values of all skin color blocks are not 0 .

\section{A. Geometric Filtration}

In addition to the facial skin, the skin color area extracted with the skin color clustering and segmentation algorithm also includes the skin of arm and shoulder, which even includes moving external surface that does not belong to human body. However, the human face has unique features, and the non-human face area can be filtered through the following principle:

(1) The human face occupies a certain proportion in the image, which is the common "three parts and five organs", calculate the size of connected area in the detected candidate area of human face, set the threshold value as $\mathrm{T}$, when the connected area is smaller than $\mathrm{T}$, it is regarded as non-human face area, and this area will be abandoned and blackened.

(2) The front human face area is close to an ellipse, and under non-extreme situation, the length-width ratios of the bounding rectangles of the profile, looking up face and looking down face are all within a certain scope. Through multiple experiments, we obtained the scope of length-width ratio is $[0.5,2.5]$, and therefore, the candidate area outside of this scope can be filtered and blackened.

(3) Because the color and skin color of eyebrows and eyes (sometimes the nostrils and mouth area) have significant difference on both chromaticity and brightness, therefore, these areas form one or more than one hole in the facial skin area. However, this kind of situation is rare in other skin area, so in accordance with the Euler 
number criterion, the non-human face area can be filtered. Set $\mathrm{E}$ as the Euler number, $\mathrm{C}$ as the number of connected area and $\mathrm{H}$ as the number of hole, then for the human face area, there is: $E=C-H \leq 1-1=0$.

The area with $E>0$ should be abandoned and blackened.

(4) The lips area is not considered for now.

In accordance with the region segmentation algorithm and the binary image after geometric filtration, see Figure 4.

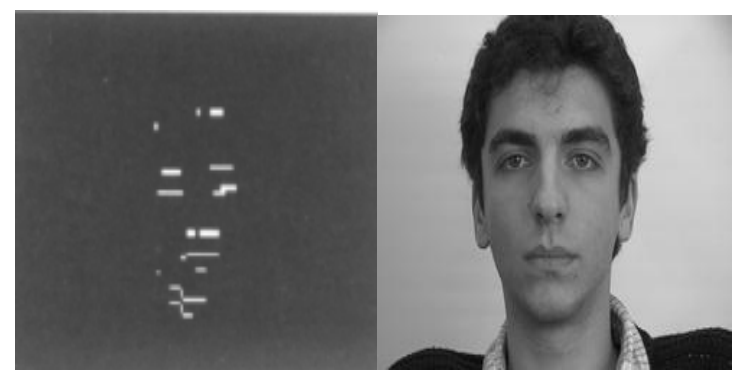

Figure 4. Binary image after region segmentation

\section{B. Coarse Positioning of Human Eyes}

The candidate areas of human face obtained above all contain a certain number of holes. Through pairing of the holes in each area, all possible two eye pairs are formed. Define a data structure set eyes $\mid$ eyes $=\{L(x, y), R(x, y)$, $\mathrm{d}, \theta\}\}$, and describe these possible two eyes. In it, $\mathrm{d}$ refers to the distance between two holes, $\theta$ refers to the included angle between the line linking the two holes and the horizontal axis, in the meantime, this angle is also the inclination angle of human face, and $\mathrm{L}$ and $\mathrm{R}$ refer to the centroid coordinates of the two hole areas respectively. The centroid coordinate is calculated in accordance with $\bar{x}=\frac{\sum_{i=0}^{n=1} \sum_{j=0}^{m=l} j B[i, j]}{A}, \bar{y}=\frac{\sum_{i=0}^{n=1} \sum_{j=0}^{m=l} i B[i, j]}{A}$, the following formula, in which, $A=\sum_{i=0}^{n=1} \sum_{j=0}^{m=l} B[i, j]$ refers to the area of hole.

Search each candidate area of human face respectively, find all possible two eye pairs with $|\theta|<45^{\circ}$ and $L(x)<R(x)$, and add them to the above set. Here, for the defined size, it is considered that the inclination angle of driver's face captured by the camera won't be too big, and the front image is preferred to reduce the computation overhead.

\section{AcCurate Positioning Algorithm OF Human EYES}

Through the above process, the real human face area has been found, and the approximate position of two eyes is found during positioning of the five sense organs. In the following, a new Hough transformation ellipse detection algorithm will be used to accurately and dynamically generate the whole curve shape of eyes.

\section{A. Human Eye Model}

At present, related positioning methods for human eyes select the eyeball center or the iris center as the positioning point, and see Figure 4 for the eyeball structure. Eyeball is a sphere with a radius of $\mathrm{R}$ : the iris is at the front of eyeball with a radius of $r$; the distance between the eyeball center and the iris center is d, and there is $\mathrm{R}^{2}=\mathrm{r}^{2}+\mathrm{d}^{2}$. In accordance with the description in literature [13], the radiuses of the eyeball and iris are constant values, and $\mathrm{R} / \mathrm{r}$ is also a constant. Therefore, we only need to measure the radius of iris $-r$, and we can obtain $\mathrm{R}$ and $\mathrm{d}$. The front structure of eye is defined as the spinning cone shape, the middle part is iris, the endpoints at two sides are the interior angle point and exterior angle point respectively, and the upper and lower arcs refer to the upper eyelid and lower eyelid respectively, as shown in Figure 5.

In our algorithm, the interior angle point, exterior angle point, upper eyelid, lower eyelid and the centroid point obtained through the above coarse positioning of human eye area are used to draw the longitudinal section of ellipse, so it does not need to conduct accurate positioning of eyeball or iris. First of all, the 5 parameters of the ellipse are analyzed, and the Hough transformation ellipse detection algorithm is used to generate the eye curve and accurately position the area of human eyes.

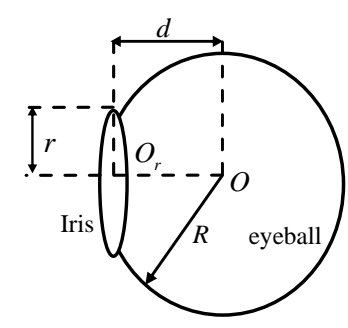

Figure 5. Eyeball structure

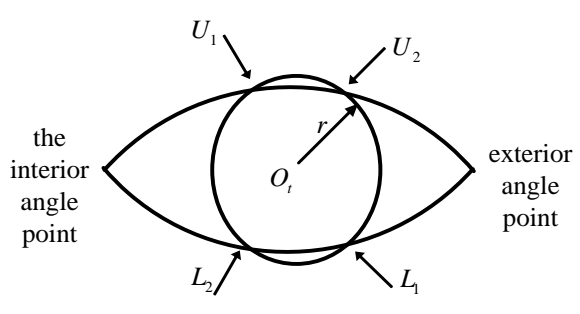

Figure 6. Spinning cone-shape eye model

\section{B. Ellipse Detection Algorithm}

The ellipse detection algorithm has always been a key issue during image processing, and just due to this reason, there are various ellipse detection methods now. Because it requires 5 parameters to completely define an ellipse, therefore, it requires a five-dimensional parameter space to detect an ellipse, which is a very time consuming work. Literature [14] used a new ellipse detection algorithm, which uses the long axis of ellipse to rapidly and effectively find the parameters of ellipse, and it only needs a one-dimensional accumulative array to accumulate the lengths of the short axes of ellipse. In this way, the required computation storage space is much 
smaller than the previous algorithms. We used the method of Literature [14] into our system.

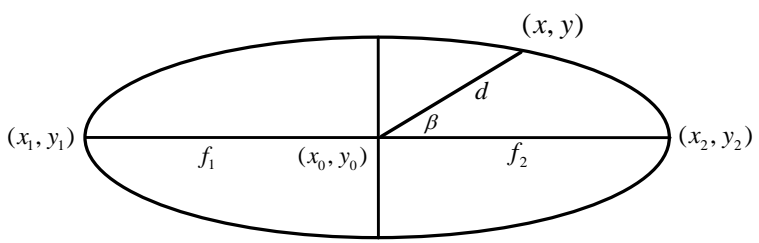

Figure 7. Geometrical characteristic of ellipse

In the ellipse in Figure 6, there are 5 ellipse parameters: the centroid point $(x 0, y 0)$, the ellipse direction angle $\alpha$ and the lengths of long axis and short axis $(a, b)$. We add addition information to each boundary point or use special boundary point, and in this way, we only need a small amount of boundary points to determine the position of an ellipse. The interior angle point $(x 1, y 1)$ and exterior angle point $(x 2, y 2)$ represent the two endpoints of long axis (which can only be the two endpoints of long axis). The 4 parameters of ellipse can be calculated in accordance with the following method:

$$
\begin{gathered}
x 0=\frac{x 1+x 2}{2} \\
y 0=\frac{y 1+y 2}{2} \\
a=\frac{\sqrt{(x 2-x 1)^{2}+(y 2-y 1)^{2}}}{2} \\
\alpha=\arctan \frac{y 2-y 1}{x 2-x 1}
\end{gathered}
$$

Assume $\mathrm{f} 1$ and $\mathrm{f} 2$ are the two foci of ellipse, $(x, y)$ is a random third point on the ellipse, and we can use $(x, y)$ to calculate the fifth parameter of this ellipse. Apparently, the distance between $(x, y)$ and $(x 0, y 0)$ is smaller than the distance between $(x 1, y 1)$ and $(x 0, y 0)$ or the distance between $(x 2, y 2)$ and $(x 0, y 0)$.

In this way, the computational formula for short axis is:

$$
b^{2}=\frac{a^{2} d^{2} \sin ^{2} \beta}{a^{2}-d^{2} \cos ^{2} \beta}
$$

In which, $\cos \beta=\frac{a^{2}+d^{2}-f^{2}}{2 a d}, d$ refers to the distance between $(x, y)$ and $(x 0, y 0)$, and $\beta$ represents the included angle between $(x, y)$ and $(x 0, y 0)$.

\section{Hough Transformation Ellipse Detection}

Hough transformation is a very effective shape analysis method, which is insensitive to stochastic noise, and it has been widely used in detection of straight line, circle and ellipse. Its basic idea is to transform the spatial domain of image to the parameter space and use a certain parameter form that satisfies most boundary points for description.

Curve on the image (area boundary). The Hough transformation detection technique calculates the parameters of boundary curve in accordance with local measurement, so it has great fault tolerance and robustness to the interruption of area boundary caused by noise interference or being covered by other target [15].

The general form to express the parameters of Hough transformation analytic curve is:

$$
f=(\vec{X}, \vec{a})=0
$$

At this moment, point $\vec{X}=[x, y]^{T}$ is a point on the ellipse, and point $\vec{a}=[d, \beta, b]^{T}$ corresponds to the parameter of ellipse. The ellipse of image space corresponds to one point in the parameter space $(d, \beta, b)$. A set point $(x, y)$ restrains a group of parameters $(d, \beta, b)$ of ellipse that pass through this point, which is equivalent to restraining the track of point $(d, \beta)$ that generates a series of ellipses. When point $(x, y)$ moves along this series of ellipses in the image space, for each point on the ellipses boundary, the parameter variation in corresponding parameter space forms a spinning cone track. This is consistent with the human eye model described in Section IV. Conduct appropriate quantification to the parameter space of Hough transformation, a three-dimensional accumulator array is obtained, and in the array, each small cubic lattice corresponds to the discrete value of parameter $(d, \beta, b)$.

Through the above ellipse detection algorithm, accurate positioning of human eyes can be rapidly and stably conduced based on detection of human face. The specific realization measures are as the following:

(1) For the color image, first of all, transform the image into grey-scale image in accordance with the color space of skin color clustering.

(2) Then, the grayscale image can be further transformed into a binary image by selecting appropriate threshold value.

(3) In accordance with the connectivity of human face and the clustering feature of skin color, the skin color clustering and segmentation algorithm in this paper is used to conduct region segmentation of human face, then, geometric filtration is conducted to the segmentation image, and the centroid point of hole in the candidate human face area is calculated to find possible human eye pair.

(4) In this paper, the Hough transformation ellipse detection algorithm is used to accurately position the human face. Conduct shape detection to the ellipse boundary existing in the image space, firstly, calculate the gradient information of the intensity of each point on the image, then, obtain the edge in accordance with appropriate threshold value, and then add 1 to the accumulator of edge $(d, \beta, b)$ and small cubic lattice. 


\section{EXPERIMENT RESULT}

For the Hough transformation ellipse detection algorithm to accurately position the curve of human eyes based on the skin color clustering space, we selected 50 images from the JAFFE (Japanese Female Facial Expression) database [10] of Japanese Kyushu University (200 images) and the Internet (300 images) respectively to test the human eye detection of different images of human face. For the standard human face database, due to the simple background and standard posture, it can rapidly find the position of human eyes with this algorithm; for the latter with multiple postures and backgrounds, some even have certain noise disturbance, this algorithm can also accurately position human eyes, but there exits certain pixel error, and the test method is as the following:

First of all, find the centroid point of human eye pair in the binary image in Figure 3, as shown in Figure 8.

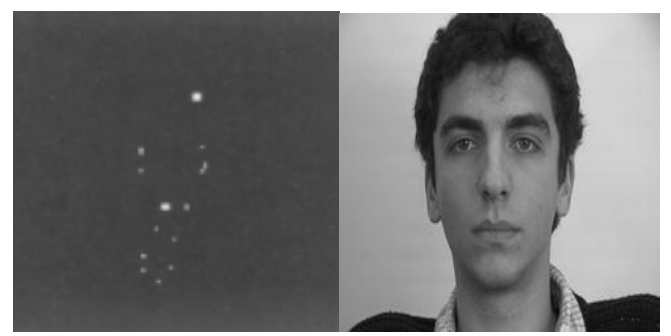

Figure 8. Centroid point of characteristic part of human face

First of all, manually find the coordinates $L(x, y)$ and $R(x, y)$ of the centroid points of the left and right human eyes, then, find the interior angle point and exterior angle point of human eye area in the binary image in Figure 3, and conduct accurate positioning of the profile curve of human eyes with the algorithm in this paper. Then, manually find the actual positional coordinates of the extreme points of upper and lower eyelids (take the left eye for example) $A_{u p}\left(x_{u p}, y_{u p}\right)$ and $B_{d o w n}\left(x_{d o w n}, y_{d o w n}\right)$, then, on the accurate positioning image of human eyes in Figure 8 obtained with this algorithm, find the computation positions of the extreme points of upper and lower eyelids $A_{u p}\left(x_{u p}, y_{u p}\right)$ and $B_{\text {down }}\left(x_{\text {down }}, y_{\text {down }}\right)$, calculate the Euclidean distances between them- $-\mathrm{d}$ and $\mathrm{d}$ respectively, and then calculate the relative errors:
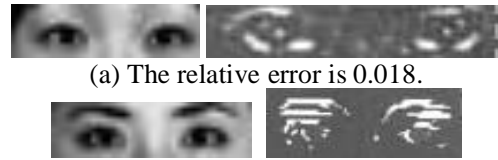

(b) The relative error is 0.041 .

Figure 9. Sample diagram of the test result from the JAFFE human face database

By making use of the data obtained from the test, the relative error curve can be drawn, which is shown in the Figure The ordinate represents the relative error $d$ (eye) values, and the abscissa refers to the percentage (\%) of eye image number tested in corresponding relative error $\mathrm{d}$ (eye) among the total number of images (500).

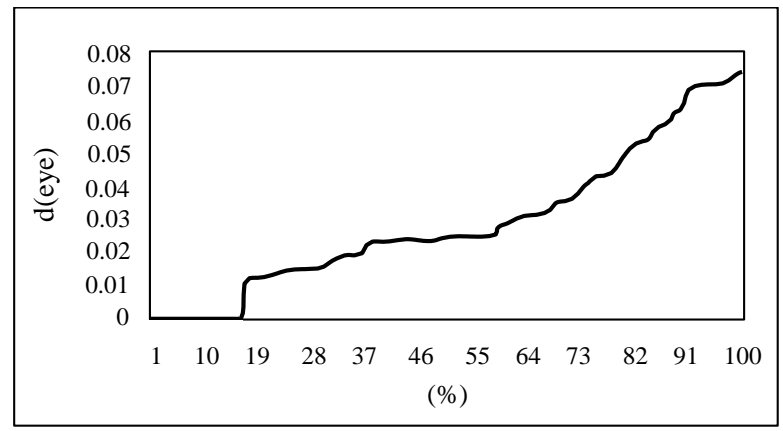

Figure 10. The relative error curve

Several experimental result figures are shown as in Fig. 9, some of which belong to pictures of daily life. (a) refers to the original image, (b) shows the skin color similarity image, (c) is the binary image after morphological filter processing, and (d) represents the image of human face and eye positioning results (the human face is marked with the green frame, and the human eyes are marked with the red frame).

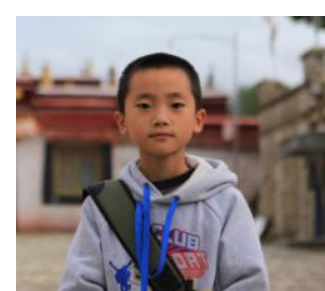

(a)

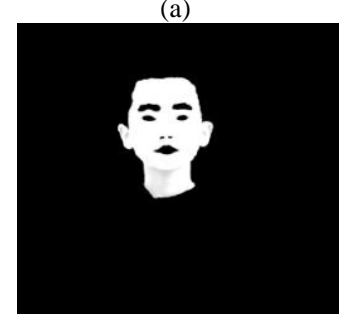

(c)

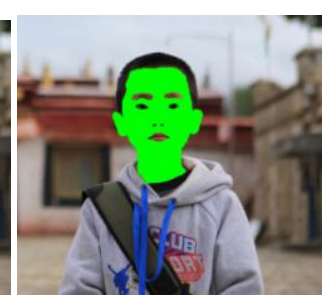

(b)

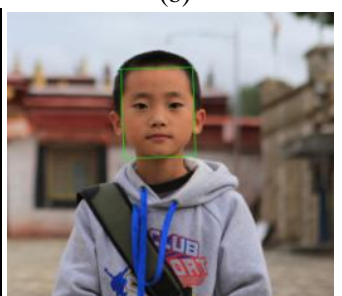

(d)
Figure 11. Execution process of the test result from a complicated background

Table 1 has listed the execution time comparison of each step of the precise positioning fig.11 (a) (d)of this algorithm and the relevant algorithm (operating in a computer with the CPU frequency of $1.8 \mathrm{GHz}$ ).

TABLE I. EXECUTION TIME COMPARISON OF EACH STEP OF THE PRECISE POSITIONING FIGURE 11 (A) OF THIS ALGORITHM AND THE RELEVANT ALGORITHM

\begin{tabular}{|l|l|l|l|}
\hline & $\begin{array}{l}\text { Positioning of the } \\
\text { true human face } \\
\text { region (ms) }\end{array}$ & $\begin{array}{l}\text { Precise eye } \\
\text { positioning } \\
(\mathrm{ms})\end{array}$ & $\begin{array}{l}\text { Total } \\
\text { execution } \\
\text { time (ms) }\end{array}$ \\
\hline $\begin{array}{l}\text { Literature [24] } \\
\text { method }\end{array}$ & 92 & 289 & 381 \\
\hline $\begin{array}{l}\text { Literature [13] } \\
\text { Hough } \\
\text { transformation } \\
\text { method }\end{array}$ & 125 & 388 & 513 \\
\hline $\begin{array}{l}\text { Method of this } \\
\text { paper }\end{array}$ & 68 & 208 & 276 \\
\hline
\end{tabular}

In accordance with the data of test results, we can see that the above algorithm can be used to rapidly and 
accurately position human eyes during human face detection. During the test to accurately position human eyes with the Hough ellipse detection algorithm, the maximum relative error $\mathrm{d}($ eye) between the obtained extreme values of upper and lower eyelids and the actual position is 0.104 . In other words, among the 50 images used for test, within the relative error scope of 0.104 , it is regarded that human eyes have been accurately positioned, and the accuracy of this algorithm can reach 0.104. In the meantime, during the test to accurately position human eyes with the algorithm proposed in this paper in a complicated background, we find this algorithm can also conduct rapid and accurate positioning. We can see the algorithm proposed in this paper has a low time complexity.

As can be seen from Table 1, the algorithm provided by this paper requires less time for the positioning of true human face region and precise eye positioning compared with the literature [24] method and the literature [13] Hough transformation method, the increased rate of total execution time is by $38.04 \%$ and $98.9 \%$ respectively. Wherein, the average computation time refers to the mean obtained by 10 times of algorithm. Through contrast, it can be seen that the computing result of Hough transformation of this paper is basically in consistency with the classical Hough transformation calculation, but the speed of the former has been doubly increased, which is significant. This is mainly because 5 parameters are required to be tested by the classical Hough transformation ellipse test method. The calculated amount of it is $(n * n)^{5}$, in which, $\mathrm{n}$ refers to the size of image. However, the amount of the algorithm provided by this paper is only $\left(n^{*} n\right)^{3}$. The larger the image is, the more ellipses shall be detected, and the higher the efficiency of this algorithm will be.

\section{CONCLUSION}

Fatigue driving has always been a main cause of car accidents, so it has important realistic significance to detect the driver's fatigue state and reduce the accidents caused by fatigue driving. Positioning of human eyes is the precondition to build the detection system of fatigue driving. In this paper, an innovative method for accurate positioning of human eyes based on the color space of skin color clustering and Hough transformation ellipse detection is proposed, in other words, the region segmentation method based on the clustering color space is used to conduct coarse positioning of eyes first, and then, the accurate positioning of eyes based on the Hough transformation of ellipse detection is conducted. This can significantly improve the computation speed of eye positioning, which can also increase the accuracy and robustness of positioning. How to conduct fatigue test to the already accurately positioned human eyes is our next research task.

\section{ACKNOWLEDGMENT}

This research is partially supported by the Science and Technology Planning Project of Hunan Province (No.
2012GK3096,No.2012FJ4334); the Natural Science Foundation of Hunan Province of China (No.12JJ6057); and Scientific Research Fund of Hunan Provincial Education Department (No. 12C1158, No. 13B132, No. 10C0374,NO.13C613 and NO. 13C614).

\section{REFERENCES}

[1] M. Reinders, R. Koch, and J. Gerbrands, "Locating facial features in image sequences using neural networks," $F G$, pp. 230-235, 1996.

[2] Z. W. Zhu, K. K. Fujimura, and Q. Ji, "Real-time eye detection and tracking under various light conditions," in Proceedings of ACM SIGCHI Symposium on Eye Tracking Research and Applications, New Orleams: Academic, 2002, pp. 139-144.

[3] X. Liu, F. Xu, and K. Fujimura, "Real-time eye detection and tracking for driver observation under various light conditions," in IEEE Intelligent Vehicle Symposium, Versailles: Academic, 2001, pp. 18-20.

[4] J. Bala, K. DeJong, and J. Huang, Visual routine for eye detection using hybrid genetic archtectures," in Proceedings of the $13^{\text {th }}$ International Conference on Pattern Recognition, E. Backer and E. Gelsema, Eds. Los Alamitos: IEEE CS Press,1996, pp. 606-610.

[5] G. C. Feng, P. C. Yuen, "Multi cues eye detection on gray intensity image," Pattern recognition, vol. 34, pp. 10031046, May 2001.

[6] J. Huang, D. Li, and X. Shao, "Pose discrimination and eye detection using support vector machines(svms)," in Proceeding of NATO-ASI on Face Recognition: From Theory to Application, 1998, pp.528-536.

[7] C. Wenming and F. Hao, "Study of an algorithm for face pose adjustment based on eye location," in Proceedings of the $5^{\text {th }}$ World Congress on Intelligent Vontrol and Automation, 2004, pp.4190-4194.

[8] J. Zhang, X. F. Yang, and R. L. Zhao, "Eye Detection Based on Hough Transform," Journal of Computer Engineering and Application, vol. 27, pp. 43-71, 2005.

[9] X. H. Sun, G. Y. Chen, C. X. Zhao, and J. Y. Yang, "Gaze Estimation of Human Eye Based on Hough Transform and Gradient Information," Journal of Chinese Computer Systems, vol. 6, pp. 1123-1128, 2007.

[10] L. Guo, Z. S. Tang, "Specification and verification of the triple-modular redundancy fault-tolerant system," Journal of Software, vol. 14, pp. 28-35, Jan 2003.

[11] S. K. Singh and D. S. Chauhan, "A robust skin color based face detection algorithm," Tamkang Journal of Scienc and Engineering, vol. 6, pp. 227-234, April 2003.

[12] T. Kanungo, D. M. Mount, and N. S. Netanyahu, "An efficient K-means clustering algorithm: analysis and implementation," IEEE Transactions on Pattern Analysis and Machine Intelligence, vol. 24, pp. 881-892, July 2002.

[13] Z. H. Zhou and X. Geng, "Projection functions for eye detection," Pattern Recongnition, vol. 37, pp. 1049-1056, May 2004.

[14] J. G. Wang, S. Eric, and V. Ronda, "Eye gaze estimation from a single image of one eye," in Proceedings of the 9th IEEE International Conference on Computer Vision Nice, France: Academic, 2003, pp. 136-143.

[15] T. Q. Wang, G. F. Xing, and B. Jiang, "Algorithm for Detection and Locating Human Face Based on Region Segmenation in Complex Background," Computer Engineering and Degisn, vol. 11, pp. 2090-2092, 2004.

[16] W. Zhong, Z. M. Liu, and J. L. Zhou, "Study on Precise Eyes Location in Face Detection," Journal of Computer Engineering and Application, vol. 36, pp. 73-76, 2004. 
[17] G. S. Xiang,X. Y. Wang, and D. T. Liang, "Real-time detection and tracking algorithm based on the color and character of human face," Opto-Electronic Engineering, vol. 4, pp. 44-48, 2007.

[18] V. Hemige, "Object-Oriented design of the groupware layer for the ecosystem information system," University of Montana, 1995.

[19] A. Rose, M. Perez, and P. Clements, Modechart toolset user's guide, Technical Report, NML/MRL/5540-94-7427, Austin: University of Texas at Austin, 1994.

[20] Bing Feng, Xiao Qing Ding, Off-line handwritten Chinese character recognition with hidden Markov models, in: 5 th International Conference on Signal Processing Proceedings, WCCC-ICSP 2000, vol. 3, pp. 1542-1545.

[21] A.V. Nefian, Embedded Bayesian networks for face recognition, Proc. of the IEEE International Conference on Multimedia and Expo, Vol. 2, 26-29 August 2002, Lusanne, Switzerland, pp. 133-136

[22] Petar S. Aleksic, Member, IEEE, and Aggelos K. Katsaggelos, Fellow, IEEE. Automatic Facial Expression Recognition Using Facial Animation Parameters and Multistream HMMs. IEEE Transactions on Information Forensics and Security, Vol. 1, No. 1, pp. 3-11, March. 2006.

[23] Michael J L, Julien Budynek, Shigeru A kamatsu. Automatic Classification of Single Facial Images. IEEE Transactions on Pattern Analysis and Machine Intelligence 1999, 21 (12) pp. 1357-1362.

[24] Zhong Wei, Liu Zhiming, Zhou Jiliu.Study on Precise Eyes Location in Face Detection. Computer Engineering and Applications, 2004, 36, pp. 72-76.

Qiufen Yang received her B. Eng. and M. Eng. degrees degree from Nanjing University, Jiangsu, China and National Defense Science and Technology University, ChangSha, Hunan, China in 1996 and 2004, respectively. She is currently pursuing her $\mathrm{Ph}$ D. degree in the School of software, Central South University,
Changsha, Hunan, China. Her current research interests are in the areas of data fusion and computer vision.

Huosheng $\mathrm{Hu}$ received his $\mathrm{Ph}$. D. degree from Oxford University, UK. He is a Professorin Computer Science at the University of Essex, UK, leading the Human Centred Robotics Group. His research interests include biologically inspired robotics, service robots, human- robot interaction, evolutionary robotics, data fusion, artificial life, embedded systems, pervasive computing and RoboCup. He is also a Chartered Engineer, a senior member of IEEE and a member of IEE, AAAI, IAS, IASTED and ACM.

Weihua Gui received the degree of the B. Eng. (Automatic Control Engineering) and the M. Eng. (Control Science and Engineering) from Central South University, Changsha, China in 1976 and 1981, respectively. From 1986 to 1988 he was a visiting scholar at Universität-GH-Duisburg, Germany. He has been a cademician of Chinese Academy of Engineering, a full professor in the School of Information Science \& Engineering, Central South University, Changsha, China, since 1991. His main research interests are in modeling and optimal control of complex industrial process, distributed robust control, and fault diagnoses

Shu-Ren Zhou eceived his PhD in 2009 from Central South University, China. He received his MS and BS in 2004 and 1999 respectively from the Changsha University of Science\& Technology and Central South University, China. His currently a post-doctoral at the National University of Defense Technology. Hiscurrent research interests include image processing, pattern recognition, human pose estimation and computer vision.

Can Zhu received his $\mathrm{Ph}$. D. in Computer Application from Central South University, China, in 2009. He is engaged as a lectorate of Changsha University of Science \& Technology, China. His research interests involve modeling and optimization concerned transportation planning and management. 